\title{
Linx
}

Revue des linguistes de l'université Paris X Nanterre

$53 \mid 2005$

Le semi-figement

\section{La préposition par, génératrice de polylexicalité ?*}

The preposition PAR, a polylexicality-maker?

\section{Badreddine Hamma}

\section{OpenEdition}

\section{Journals}

Édition électronique

URL : http://journals.openedition.org/linx/262

DOI : $10.4000 /$ linx.262

ISSN : 2118-9692

\section{Éditeur}

Presses universitaires de Paris Nanterre

\section{Édition imprimée}

Date de publication : 1 décembre 2005

Pagination : 87-102

ISSN : 0246-8743

\section{Référence électronique}

Badreddine Hamma, «La préposition par, génératrice de polylexicalité ?*», Linx [En ligne], 53 | 2005,

mis en ligne le 11 février 2011, consulté le 01 mai 2019. URL : http://journals.openedition.org/linx/262 ; DOI : 10.4000/linx.262 


\title{
La préposition par, génératrice de polylexicalité ?**
}

\author{
Badreddine Hamma \\ Université de Paris $X$ - Nanterre et Laboratoire MoDyCo \\ (CNRS UMR 7114)
}

\section{Introduction}

Le problème d'exhaustivité que rencontrent les dictionnaires quant à l'inventaire de différentes sortes de collocation (locutions adverbiales, prépositives, nominales et expressions figées), ainsi que l'hésitation dont témoigne la disparité des gloses données à leur sujet, çà et là - lacunes relevées déjà par S. Mejri (2002) -, s'expliquent sans aucun doute par le fait que l'intérêt porté à la polylexicalité dans les études linguistiques est relativement récent. Longtemps les collocations ont été contournées dans l'étude du lexique, car considérées comme non représentatives de l'usage standard, ordinaire, de la langue, et donc marginales. Mais cet intérêt tardif que l'on porte à l'étude du figement, à la polylexicalité, aux proverbes, etc. n'implique pas que le phénomène langagier vienne d'apparaitre. $\mathrm{Si}$, théoriquement, la composition lexicale et le figement viennent, du point de vue chronologique, après la base monomorphémique dont ils tirent leur existence, ils ne relèvent pas, comme le montre S. Mejri (2002), d'un fait accidentel ou d'une quelconque forme d'intrusion; ils font partie intégrante du système linguistique de toute langue naturelle; de fait, quand ils

\footnotetext{
* Je tiens à remercier mes relecteurs anonymes pour leurs remarques judicieuses et enrichissantes.
} 
sont marginalisés, la description que l'on fait de la langue reste partielle. C'est alors que de nouvelles perspectives sont ouvertes et de nouvelles mesures sont prises dans le domaine de la recherche sur le lexique visant à pallier les lacunes existantes. C'est dans cette perspective que s'inscrit le présent travail : à l'issue d'une étude où nous avons essayé d'établir l'invariant sémantique de la préposition par, sous-jacent à la diversité de ses emplois dans le discours, nous avons découvert que, dans un grand nombre d'énoncés, par entretient un rapport privilégié avec son entour: les paradigmes lexicaux sélectionnés par la préposition sont, d'une part, récurrents tels quels, d'autre part, résistent à certaines opérations linguistiques (ils donnent lieu à des énoncés agrammaticaux ou à un important changement de sens). Cette découverte a été possible grâce à l'observation d'un large corpus renfermant différentes actualisations de par, ce qui a permis de relever une construction assez productive d'unités polylexicales (voir annexe, pour la liste des unités établie) formées selon le schéma $P A R+S N$, avec des $N$ sans déterminants : par hasard, par chance, par accident, par exemple, par cour, par inadvertance, par effluves - certains de ces $\mathrm{N}$ sous-catégorisent une modification de type adnominal : par opposition à N, par comparaison avec $N$, par considération pour $N$, etc. -, ou avec des $N$ actualisés qui sont parfois modifiés (par la force des choses, par l'absurde, par les temps qui courent, par la petite porte, par l'intermédiaire de N, par le biais de N, par l'entremise de N, etc.). Ces différentes collocations se distinguent d'autres ayant globalement le même schéma syntaxique, mais qui relèvent a priori de constructions libres : par la fenêtre, par Paul, par jalousie, par ma voisine, etc., contrairement aux premières qui tendent vers le figement.

Dans le présent travail, nous nous proposons d'appréhender les collocations en par qui manifestent certaines caractéristiques empiriques de figement : l'application des tests et des critères dévolus à la reconnaissance des séquences (semi-)figées est vérifiée avec ces collocations. Nous essaierons, en particulier, de classer les séquences relevées en fonction de leur valeur grammaticale, leur réaction aux différentes manipulations linguistiques et, ipso facto, leur degré de figement. Nous montrerons que la formation des unités polylexicales en par répond à deux besoins linguistiques incontournables : un besoin morphologique (la construction d'une unité à valeur adverbiale à partir d'un nom, par exemple, est problématique, étant donné que le suffixe -ment ne se greffe que sur des adjectifs) et un besoin sémantique (même dans le cas où la formation serait possible avec un adverbe en -ment, le sens obtenu ne tient pas compte d'un effet de sens important dans une séquence en par $(+\mathrm{N})$, à savoir la valeur de «modalité contrastive ».

\section{La valeur grammaticale des unités polylexicales en par}

Parmi les séquences polylexicales ${ }^{1}$ où par est en vedette, on peut déceler des combinaisons susceptibles de fonctionner comme des "structures en interposition" (L. Melis 2004 : 22) qui ont la valeur d'un adverbe de manière (un par un, sou par sou, cas

\footnotetext{
${ }^{1}$ Les mots polylexicaux, appelés aussi « complexes », selon G. Gross (1996), sont des unités composées d'au moins deux mots simples (y compris par dérivation, i.e. portemanteau, porte-clés, etc.) ; ce phénomène concerne des suites du type boule de billard; les carottes sont cuites; avoir la frite/ avoir la dent; etc., mais non (la) boule de Paul; Les frites sont cuites; avoir les/la carotte(s)/ avoir mal aux dents; etc. - ces dernières sont analysables comme des constructions libres.
} 
par cas, etc.) $)^{2}$, des locutions adverbiales (par hasard, par contre, par bonbeur, par trop, etc.), des locutions prépositives (de par, par chez, par lintermédiaire de, etc.), des périphrases verbales qui ont, globalement, les mêmes propriétés linguistiques que les semi-auxiliaires inventoriés dans les ouvrages d'usage (commencer par, finir par $+\mathrm{V}$ à l'infinitif et, de façon moins fréquente, débuter par, couronner par et terminer par $+\mathrm{V}$ à l'infinitif) ${ }^{3}$, mais aussi des tournures phrastiques où la présence de par est significative (se tenir par la barbichette, parler par la bouche de qqn, être/ aller par monts et par vaux, entrer par la grande porte, entrer par la petite porte, manger les pissenlits par la racine, etc.). Dans cette étude, nous nous focalisons sur celles qui fonctionnent comme des locutions adverbiales ou prépositives et dont l'inventaire donné traditionnellement ne rend guère compte.

Dans les descriptions lexicographiques disponibles, certaines collocations en par sont citées seulement comme des associations libres typiques. Ainsi, le PRLF glose la séquence par l'intermédiaire de comme une «locution courante», sans pour autant préciser la valeur grammaticale qu'elle occupe dans la phrase, et dans sa définition de la notion de «locution courante » stipule : «insiste sur le fait qu'un sens, un emploi est connu et employé de tous »; or, il est aisé de constater que cette séquence a un fonctionnement stabilisé dans le système linguistique : elle régit toujours un $S N$ à la manière d'une préposition et au même titre que la locution prépositive par l'entremise de reconnue en tant que telle par le même dictionnaire. Qui plus est, cette séquence n'est pas un cas singulier ; on peut, en effet, relever d'autres séquences en par ayant le même statut grammatical et formées selon le même schéma syntaxique $(P A R+(L E+) N+$ $P R E P+S N)$ : par le canal de, par le moyen de, par comparaison avec/à, par le biais de, par le truchement de, par opposition à, par rapport à, par considération pour, par contraste avec, qui soit sont absentes dans les dictionnaires - quoique fréquentes dans l'usage -, soit sont citées comme des associations libres, ce qui est préjudiciable : cela ne prévient pas l'usager que telle ou telle séquence n'admet pas les mêmes fonctionnements linguistiques qu'une association libre correspondant au même schéma syntaxique.

On peut relever également des séquences en par manifestant les mêmes fonctionnements que les locutions adverbiales enregistrées. Dans les dictionnaires, on mentionne généralement quelques cas comme par contre, par hasard, par ailleurs, par bonbeur, par à-coups, etc.; mais sont susceptibles de la même analyse d'autres associations comme par paliers, par parenthèse, par ordre, par étape(s), par enchantement, par effluves, par ondes, par bouffées, par écrit, par éclairs, par degré(s), par saccades, par secousses, par intervalle(s), par le siège (accouchement), par vagues, par un malheureux / beureux concours des circonstances, par ricochet, par la bande, etc., qui, contrairement au premier type de locutions (celles identifiées comme des locutions prépositives), ne sont pas suivies d'un $S N$ et peuvent être paraphrasées par un adverbe en -ment et/ou par une tournure du type « de façon/manière + un adjectif (qui peut être morphologiquement apparenté au N) » :

1. Par paliers/vagues/effluves/étapes/ordre/bouffées/ondes $=>$ « de manière/façon progressive »/ «progressivement»

${ }^{2}$ Cf. B. Hamma (2005b).

${ }^{3}$ Cf. B. Hamma (2004a et 2004b). 
2. Par éclairs, par instant(s)/moment(s)/intermittence(s)/intervalle(s)/ secousses/ saccades => «de façon/manière irrégulière »

3. Par malheur/bonheur/un malheureux/heureux concours de circonstances $=>$ « (mal)heureusement »/ «se façon (mal)heureuse »

L'existence de telles occurrences incite d'une part à en étudier le corrélat sémantique ainsi que les caractéristiques empiriques permettant d'asserter que nous avons affaire à des cas de (semi-) figement et d'autre part à en délimiter la portée.

\section{Degré et limites du figement des collocations en par}

\subsection{Approche sémantique}

Dans un premier temps, il faut rappeler l'identité sémantique de la préposition par telle que nous l'avons établie (Hamma 2005a) à partir de l'étude d'un grand corpus de données. Cela permet de mieux comprendre les fonctionnements des collocations où elle occupe un statut privilégié. Par la suite, nous nous penchons sur l'interaction des différents constituants des polylexicalités prises en compte.

\subsubsection{L'invariant sémantique de la préposition par ${ }^{4}$}

La préposition par se rattache à une construction élémentaire décelable dans n’importe quel énoncé : par, son régime (par $\mathrm{R})$ et un procès $(P)$ véhiculé par le verbe, qui peut ne pas apparaitre en surface mais que l'on peut reconstituer à partir de paraphrases particulières ou par induction ${ }^{5}$. Ainsi, la relation $(P)$ par $R$ se présente comme une structure de base irréductible sous-jacente à l'emploi de par dans toutes ses facettes discursives. Cette régularité au niveau de la construction conduit à y discerner une régularité sémantique, une invariance, conformément au "principe de naturalité » qui sous-tend notre démarche (Milner 1989 : 307) : à une forme invariante correspond un sens invariant (de même qu'une différence de forme en appelle une sur le plan sémantique et vice versa). Selon nos principes heuristiques, par $\mathrm{R}$ correspond à une " modalité » qui conditionne le déroulement du procès $(P)$ et garantit son aboutissement à un certain résultat final. Ainsi dans les constructions libres suivantes (relevant de valeurs discursives différentes) :

1. On y accédait par un couloir, interdit aux chats, où s'amoncelaient des tableaux.

2. Le pont a été détruit par la tempête.

\footnotetext{
${ }^{4}$ Ici, nous reprenons uniquement les éléments les plus significatifs de l'invariant sémantique de la préposition par qui nous serviront dans la suite de l'exposé (pour les détails se reporter à Hamma 2005a).

5 Ainsi, dans l'emploi de par dit d'« agent» (L'Education sentimentale par Flaubert), on a un procès non exprimé explicitement, du type écrit, créé, conçu, etc. comme pourrait le confirmer le rapport stéréotypique que l'on peut établir entre Flaubert (« romancier») et L'Education sentimentale (« roman »).
} 
La préposition par, génératrice de polylexicalité?

3. On a constaté que pour transporter des glaces par mer il fallait parfois opérer en caisses étanches soudées.

4. Ensuite vous roulez la pâte par petites bandes, que vous coupez de la grosseur d'une aveline, en les roulant très rondes et en les saupoudrant légèrement de farine.

5. Les procès-verbaux sont remis ou envoyés par lettre recommandée directement au Procureur de la République.

Les procès dénotés par les formes verbales, telles que réalisées dans ces énoncés, ont une valeur perfective ${ }^{6}$; ils impliquent l'aboutissement à un terme : en (4), (accéder) « quelqu'un est quelque part»; en (5), (détruire) « le pont est détruit »; en (6), (transporter) «des glaces sont transportées »; en (7), (rouler quelque chose) «la pâte est roulée »; en (8), (remettre ou envoyer) « des procès-verbaux sont remis ou envoyés », et le $S P$ en par, à chaque fois, précise la «modalité » permettant d'arriver à ce terme. En première approximation, cette notion de " modalité » qui renvoie au " mode de réalisation du procès » est très proche de la valeur dite de «manière », mais ne s'y réduit pas : pour une grande partie des données examinées, la question en Comment, utilisée dans la grammaire traditionnelle pour identifier la «manière» ou le «moyen», est souvent possible avec les emplois "spatial », « agentif », « instrumental », « temporel », « distributif» (dans certaines occurrences, on est amené à introduire quelques modifications pour que la question fonctionne convenablement) :

4a. Commenty accède-t-on ? => Par un couloir, interdit aux chats, où s'amoncelaient des tableaux.

5a. Comment le pont a-t-il été détruit? => Par la tempête.

6a. Comment transporte-t-on des glaces... ? => Par mer.

7a. Comment rouler la pâte? => Il faut la rouler par petites bandes...

8a. Comment les procès-verbaux sont-ils envoyés? => Par lettre recommandée...

Dans ce qui suit, nous verrons, d'une part, en quoi l'invariant sémantique de la préposition par (en rapport avec l'expression de la «modalité ») est utile dans l'approche des unités polylexicales considérées, et d'autre part, quel type d'entour la préposition sélectionne dans la formation des unités polylexicales.

\subsubsection{Les contraintes sémantiques}

En première approximation, les unités polylexicales considérées ne semblent pas constituer des expressions opaques, puisque chacun des éléments vedettes qui les compose (la préposition par et le paradigme de noms) connait de larges possibilités

\footnotetext{
${ }^{6}$ La valeur «perfective » de ces procès est décelable également à l'aide de la compatibilité de ces énoncés avec des tests, du type «en n temps» ou «depuis n temps» et leur incompatibilité devant des enchaînements, du type "pendant/durant $n$ temps» sans tomber dans la valeur itérative (Gosselin 1996, pp. 25 et 46).
} 
d'emplois différents où ils gardent, globalement, leurs valeurs sémantiques respectives. Prenons le cas de la locution par hasard, dans l'exemple (9):

9. J'étais debout, tenant à la main la lettre de Madeleine, quand par hasard Olivier rentra (TLF).

Nous pouvons y reconnaitre, d'un côté, quelques valeurs de par apparentées à la représentation de la « modalité »/ « mode de l'action » (cf. supra) et à la tendance de régir un $N$ sans déterminant, dans des occurrences comme : voyager par (mer + avion); tuer par balles; agir par jalousie; envoyer un message par (fax + lettre + télégramme); etc. De l'autre, la valeur du N hasard est conservée : «cas ou événement fortuit, concours de circonstances inattendu et inexplicable », ce que l'on peut retrouver dans des occurrences différentes du type : Coups tirés au basard; Au basard des rencontres; Le hasard fait bien les choses; Faire la part du basard dans une prévision; S'en remettre au basard; etc. (PRLF). D'ailleurs, le sens général du $\mathrm{N}$ hasard est maintenu même dans les termes qui lui sont morphologiquement apparentés ${ }^{7}$ :

10. Qui ne basarde rien n'a rien

11. Ce chemin hasardeux comme une passerelle sur un torrent. (PRLF)

Il en va de même pour la catégorie des locutions prépositives avérées ( $c f$. supra) : les $\mathrm{N}$ gardent fondamentalement leur signifié spécifique ; en effet le sens global de ces locutions est paraphrasable par " (faire/obtenir quelque chose) en utilisant $\mathrm{N}$ » ou « de façon indirecte »; ainsi retrouve-t-on l'expression de la «modalité » en ce qui concerne l'apport propre de la préposition par (cela répond, grosso modo, à la question Comment?) et les $\mathrm{N}$ intermédiaire, entremise, truchement, moyen, canal, biais, ici, renvoient tous, de leur côté, à leur acception la plus fréquente dans les constructions libres : «médiation (indirecte) entre deux termes » que l'on retrouve dans des énoncés libres du type : Chainons intermédiaires d'une évolution; Se servir de l'entremise d'une personne influente pour parvenir à ses fins; Il a été le fils aimé de l'Église, son truchement, celui qu'Elle chargeait d'exprimer ses pensées; Il a trouvé le moyen d'entrer sans payer; Canal d'adduction d'eau/d'amenée/de fuite; Par quel biais le prendre?/jeter un coup d'ail en biais, etc. (PRLF)

Ainsi, du point de vue compositionnel, ces premières remarques conduisent à considérer que les syntagmes prépositionnels en par ne se prêtent pas, a priori, à une étude en termes de polylexicalité et, partant, il ne serait pas ici question d'un cas de figement, mais de constructions libres. Toutefois, cette transparence sémantique n'est, en fait, que partielle ; d'une part, ces différents $\mathrm{N}$ ne commutent pas librement avec ce que les dictionnaires proposent comme leurs synonymes: on n'aura pas, par exemple, * par l'instrument de, *par l'outil de, *par le dispositif de, *par l'appareil de, etc. parallèlement aux noms intermédiaire, entremise, truchement, moyen, canal et biais; les seules commutations possibles se limiteraient a priori à celle opérée entre ces six noms, mais selon des contraintes distributionnelles particulières; par exemple, par l'entremise de et par le canal de ne semblent sous-catégoriser que des $\mathrm{N}_{\text {HUMAIN }}$, contrairement à par le biais de qui ne sous-catégorise que des N NON HUMAIN ou par l'intermédiaire de qui admet les deux types

\footnotetext{
${ }^{7}$ Voir également B. Hamma (2002), pour un cas similaire : par exemple, qui confirme cette approche compositionnelle.
} 
de distribution. D'autres collocations construites selon le même schéma présentent plus de contraintes au niveau des $\mathrm{N}$ qu'elles renferment (par la pean des fesses; par l'opération du Saint-Esprit; par tête de pipe), ce qui permet de parler à leur égard d'un degré plus élevé de figement : l'exclusivité est emportée par un $\mathrm{N}$ particulier qui ne commute avec aucun autre $\mathrm{N}$ sans changer le sens global de la séquence, et la polylexicalité subit alors un défigement; ainsi, par la peau des fesses - collocation à valeur adverbiale qui signifie "retenir quelqu'un à la dernière minute de façon brutale » n'admet pas la commutation avec d'autres "parties du corps» en conservant le sens idiomatique : *par la peau des joues, * par la peau du derrière, *par la peau du ventre, etc. où l'on bascule dans un contexte de «saisie» ou de "préhension» - on peut toujours évidemment avoir des variantes idiolectales ou sociolectales : par la peau du cou, par la peau $d u$ cul, par la pean $d u$ dos. Ce raisonnement vaut globalement pour les deux autres séquences : par l'opération du Saint-Esprit (= « de façon mystérieuse »), par tête de pipe (= " par personne ») où les $\mathrm{SN}$ le Saint-Esprit et pipe sont les seuls possibles, ce que montre l'impossibilité de leur commutation avec des $\mathrm{N}$ pouvant être perçus comme des synonymes $\left({ }^{*}\right.$ par l'opération de Dieu/Jésus/Marie/etc., *par tête de cigarette/narguilé/cigare, etc.). Et pour revenir à la locution adverbiale par hasard, on n'a pas de commutations lexicales possibles du $\mathrm{N}$ hasard en gardant le même sens ; par chance, par exemple, spécifie le fait que les circonstances de la réalisation d'un procès donné sont à la fois fortuites et heureuses, alors qu'on peut avoir avec le N hasard des contextes « catastrophistes »; avec la locution par hasard, on insiste davantage sur le caractère fortuit de l'événement que dénote le procès de l'énoncé, indépendamment de toute évaluation «heureuse » ou «malheureuse »; c'est le contexte qui indique ce genre d'information; chance dans par chance ne commute pas non plus avec ses synonymes: * ${ }^{*}$ ar fortune, *par veine, *par aubaine, * ${ }^{*}$ ar bol; etc. En revanche, dans le sens antonymique, on peut avoir par malchance, par malheur, par manque de bol-locutions qui se soumettent aussi à des contraintes spécifiques d'ordre lexicosémantique, de la même façon que par hasard et par chance.

\subsubsection{Les contraintes syntaxiques}

A l'incompatibilité du test de la commutation (critère sémantique), s'ajoutent différents critères syntaxiques permettant de traiter ces différentes collocations comme des polylexicalités et qui affectent leurs propriétés combinatoires ( $c$. G. Gross 1996 et S. Mejri 1997). En effet, la séquence par $S N$ présente des restrictions ou impossibilités au niveau de ses distributions sur plusieurs plans. Ce blocage ancre ces séquences dans un contexte de "généricité » et non de désignation ou de « dénomination » (S. Mejri 2002) : les N chance, malchance, hasard et miracle, en entrant dans une polylexicalité en par, ne renvoient plus à un épisode particulier, mais se présentent comme une « manière de procéder générique » associée à la réalisation du procès; ils servent d'aune ou de paramètre permettant de se faire une idée générale sur le type de circonstances dans lesquelles un procès donné s'est déroulé. De fait, les tests visant à les spécifier ou à les actualiser aboutissent à des énoncés inacceptables.

L'actualisation : l'insertion d'un déterminant entre par et l'un de ces $\mathrm{N}$ n'est pas naturelle, comme le montrent les phrases agrammaticales : 
Hamma Badreddine

12. Aucun des deux hommes, par (*leur $+{ }^{*} c e+{ }^{*}$ notre $+*^{*} l e+*^{*}$ un) miracle, n'avait été blessé.

13. Mais je n'ai aucune confiance et $n^{\prime}$ attends nulle constance d'affections fondées sur le caprice, sans aucun principe fixe, même si, par ( ${ }^{*}$ cette $\left.+{ }^{*} m a+{ }^{*} l a+{ }^{*} u n e\right)$ chance, les deux se trouvent avoir des dispositions communes.

14. Elle a en bean ouvrir avec précaution la barrière de bois dont l'unique gond ronillé grince toujours, son pied a beurté par (*cette $+{ }^{*}$ sa $\left.+{ }^{*} l a+{ }^{*} u n e\right)$ malchance le seau dans lequel on brasse la bouillie de son pour les poules.

15. J'ai rêvé à l'histoire du garçon hollandais qui avait découvert, un matin, par ( ${ }^{*} c e+{ }^{*}$ son + *le $+*^{*}$ ) hasard, en se promenant, un petit trou dans la digue qui protégeait son village des flots gris de la mer du Nord, un trou minuscule qui risquait d'inonder son pays de polders.

La pronominalisation est automatiquement exclue :

12a. * Aucun des deux hommes, par lui (= miracle) n'avait été blessé.

13a. *Mais je n'ai aucune confiance et n'attends nulle constance d'affections fondées sur le caprice, sans aucun principe fixe, même si, par elle (= chance), les deux se trouvent avoir des dispositions communes.

14a. *Elle a en beau ouvrir avec précaution la barrière de bois dont l'unique gond rouillé grince toujours, son pied a heurté par elle (= malchance) le seau dans lequel on brasse la bouillie de son pour les poules.

15a. *J'ai rêvé à l'bistoire du garçon hollandais qui avait découvert, un matin, par lui (= hasard), en se promenant, un petit trou dans la digue qui protégeait son village des flots gris de la mer du Nord, un trou minuscule qui risquait d'inonder son pays de polders.

L'anaphorisation du $N$ du $S N$ en par n'est possible ni par reprise pronominale, ni par reprise lexicale :

12b. * Aucun des deux hommes, par miracle, n'avait été blessé. Ce miracle/Il arrive souvent dans ce métier.

13b. *Mais je n'ai aucune confiance et n'attends nulle constance d'affections fondées sur le caprice, sans aucun principe fixe, même si, par chance, les deux se trouvent avoir des dispositions communes. Cette chance/Elle est de toute façon complètement exclue.

14b. *Elle a en beau ouvrir avec précaution la barrière de bois dont l'unique gond rouillé grince toujours, son pied a beurté par malchance le seau dans lequel on brasse la bouillie de son pour les poules. Cette malchance/Elle l'accompagne depuis qu'elle était petite.

15b. * J'ai rêvé à l'histoire du garçon hollandais qui avait découvert, un matin, par hasard, en se promenant, un petit trou dans la digue qui protégeait son village des flots gris de la mer $d u$ 
La préposition par, génératrice de polylexicalité?

Nord, un trou minuscule qui risquait d'inonder son pays de polders. Ce basard/Il était lui a changé la vie.

La relativation est également incompatible :

12c. * Aucun des deux hommes, par miracle qui arrive souvent, n'avait été blessé.

13c. * Mais je n'ai aucune confiance et n'attends nulle constance d'affections fondées sur le caprice, sans aucun principe fixe, même si, par chance, qui est exclue de toute façon, les deux se trouvent avoir des dispositions communes.

14c. *Elle a en beau ouvrir avec précaution la barrière de bois dont l'unique gond rouillé grince toujours, son pied a heurté par malchance, qui l'accompagne depuis qu'elle était petite, le seau dans lequel on brasse la bouillie de son pour les poules.

15c. * J'ai rêvé à l'bistoire du garçon hollandais qui avait découvert, un matin, par hasard auquel il ne s'attendait pas, en se promenant, un petit trou dans la digue qui protégeait son village des flots gris de la mer du Nord, un trou minuscule qui risquait d'inonder son pays de polders.

Notons cependant qu'avec le SP par hasard, un certain type de modification est envisageable, particulièrement, avec des adjectifs restrictifs antéposés: pur, curieux (précédé de un) ou le plus grand des, avec un passage du $\mathrm{N}$ hasard au pluriel ; mais même dans ce cas, le sens du SP par hasard reste générique et ne peut renvoyer à une situation spécifique. Les autres $\mathrm{N}$ ne sont pas très naturels avec ce genre de modification (?par (pur(e) + un $(e)$ curieux $/$ se + le/la plus grand(e), des chance(s)/ miracle(s)/malchance(s)) :

15d. J'ai rêvé à l'histoire du garçon hollandais qui avait découvert, un matin, par (pur + un curieux + le plus grand des) hasard(s), en se promenant, un petit trou dans la digue qui protégeait son village des flots gris de la mer du Nord, un trou minuscule qui risquait d'inonder son pays de polders.

Concernant les collocations identifiées comme des locutions prépositives, certaines opérations syntaxiques sont appelées par la nature de la construction; en effet, tous ces $\mathrm{N}$ sont déjà actualisés : ils sont introduits par l'article défini (le/la), mais aucune commutation avec un autre déterminant n'est possible (*par ce/cet(te) + un (e) (intermédiaire + entremise + truchement + moyen + canal + biais $)$ de $N)$; le pluriel est impossible (*par deux/trois + quelques/plusieurs/certain(e)s/ (intermédiaires + entremises + truchements + moyens + canaux + biais $)$ de N). De même, la pronominalisation et l'anaphorisation sont complètement exclues comme les différents tests d'insertion ou de modification : les adjectifs, les compléments de détermination et les relatives sont impossibles (par le l' (intermédiaire + entremise + truchement + moyen + canal + biais $)$ (qui est/soit) (efficace + discret/discrète + moderne + d'experts + de qualité + pour tous)) de $N)$.

En revanche, le SN en par apparait compatible avec le test du clivage - test généralement incompatible avec les expressions identifiées comme présentant un cas de figement (*C'est sa pipe qu'il a cassée; *C'est la glace qu'il a brisée; *C'est la mouche qu'il a prise ; etc.) : 
12d. C'est par miracle qu'aucun des deux hommes n'avait été blessé.

13d. C'est par chance que les deux se trouvent avoir des dispositions communes.

14d. C'est par malchance que son pied a heurté le seau dans lequel on brasse la bouillie de son pour les poules.

15e. C'est par hasard qu'il a découvert un petit trou dans la digue qui protégeait son village des flots gris de la mer du Nord...

Cette compatibilité avec le test du clivage est corrélable, selon nous, à l'une des propriétés spécifiques de la préposition par: dans B. Hamma (2005a), nous avons mis en évidence le fait que les différentes occurrences en par réalisées dans le discours contiennent déjà la trace d'une opération d'extraction en «C'est... qu... » dans leur "phrase de base »; cela est rendu sensible grâce au type d'enchaînement que le SP admet (...et non par R) : C'est par miracle qu'aucun des deux hommes n'avait été blessé et non parce que les paramètres de sécurité étaient respectés / C'est par chance que les deux avaient des dispositions communes; ils ne se connaissent même pas / C'est par malchance que son pied a heurté le seau; personne n'avait prévu que cela allait arriver; C'est par hasard qu'il a trouvé ce trou; personne ne le lui avait montrê). Ce type d'enchaînement est relié ainsi à un certain "effet de contraste » (D. Leeman 1998, pp. 20-21) : la modalité réalisée dans l'énoncé en par renvoie implicitement à d'autres modalités exclues ou auxquelles on peut s'attendre.

Toutefois, les autres locutions prépositives relevées supra, construites sans déterminants (par comparaison avec) à, par opposition à, par rapport à, par considération pour, par contraste avec) réagissent différemment vis-à-vis du test de l'actualisation ; elles admettent généralement l'insertion d'un déterminant mais subissent alors un défigement de la séquence ; on n'a plus affaire à une modalité générique, mais à une modalité spécifique (un « dispositif») admettant des précisions locales au moyen de modifications par des adjectifs ou des compléments de détermination et des relatives. Notons que dans les exemples (16-18) et (20), c'est la préposition avec (qui introduit le SN régime) qui prend la relève, à étant impossible :

16. Par une comparaison assez éloquente avec $N$

(vs) *Par comparaison assez éloquente avec/à $N$

17. Par une opposition claire et nette avec $N$

(vs) *Par opposition claire et nette avec $N$

18. Par un rapport clair et net avec $N$

(vs) *Par rapport clair et net à avec $N$

19. Par une considération particulière pour $N$

(vs) ?Par considération particulière pour $N$

20. Par un contraste clair et net avec $N$

(vs) *Par contraste clair et net avec $N$ 
Par ailleurs, les critères proposés par J.-C. Anscombre (1990, pp. 105-123) pour pouvoir parler d'article zéro sont systématiquement vérifiés avec ces dernières occurrences :

a La non-opacité sémantique : les $\mathrm{N}$ comparaison, opposition, rapport, considération et contraste gardent globalement leur sens en emploi libre.

a. La productivité : la structure en $P A R+\varnothing+N$ est productive (voir annexe).

a L'introduction d'un déterminant sans changement important de sens : à part l'effet de généricité entrainé par la construction de ces $\mathrm{N}$ sans déterminant, on a vu supra que les $\mathrm{N}$ conservent grosso modo leur sens; on est loin des occurrences comme casser sa pipe ("mourir») ou tirer le diable par la queue ("avoir à peine de quoi vivre/avoir de maigres ressources ») dont le sens n'est pas inférable de façon directe des éléments qui constituent la séquence.

Or l'article zéro est relié, d'après J.-C. Anscombre (op. cit.), à un contexte de " généricité » et de "processivité », ce que l'on peut vérifier clairement dans la locution adverbiale par exemple (B. Hamma 2002) ; en effet, le N exemple dans une occurrence comme démontrer par exemple le théorème de Pythagore (avec article zéro) renvoie à l'acte d'exemplification lui-même (d'où le caractère processif de la séquence); le sens est que le théorème de Pythagore est pris en l'occurrence comme exemple, alors que le $\mathrm{N}$ exemple dans démontrer par l'exemple le théorème de Pythagore (avec un article défini) correspond plutôt à un procédé utilisé pour la démonstration du théorème de Pythagore, donc renvoie à un dispositif précis que l'on peut, d'ailleurs, spécifier avec des modifications : démontrer par l'exemple du triangle rectangle le théorème de Pythagore, ce qui est impossible dans le premier cas * démontrer par exemple du triangle rectangle le théorème de Pythagore, d'où la généricité de la locution par exemple par opposition à la séquence par l'exemple (dénotant un sens « instrumental »).

\section{Processus de la formation des polylexicalités en par}

Il résulte de ce qui précède que les collocations en par manifestent, selon le cas, et à des degrés variés, certaines caractéristiques empiriques de figement ; on peut distinguer trois classes :

a les locutions prépositives invariables dont le régime est un $\mathrm{N}$ libre :

$\Rightarrow$ avec déterminant : par l'intermédiaire de $N$, par le canal de N, par le biais de N, par le truchement de $N$, etc.

$\Rightarrow$ sans déterminant (avec un cas d'article zéro) : par opposition à N, par contraste avec $N$, par comparaison àl avec $N$, par considération pour $N$, etc.

a les locutions prépositives invariables dont le $\mathrm{N}$ régime est figé : par la peau des fesses, par l'opération du Saint-Esprit, par tête de pipe, etc.

a les locutions adverbiales : par miracle, par malchance, par hasard, etc.

Ce que ces locutions de différentes formations ont en commun est le fait que le SP en par (soit une locution adverbiale $P A R N$ : par hasard, par contumace, etc., soit une locution prépositive suivie d'un complément régime $P A R+N+P R E P+N$ : par l'intermédiaire de $N$, par opposition à $N$, par considération pour $N$, etc.) prend, dans son inté- 
gralité, une valeur adverbiale selon le principe de «transfert catégoriel » (cf. S. Mejri 2004) ; on peut, en effet, lui substituer, par exemple, une tournure au gérondif « en utilisant $\mathrm{N}$ » ou des paraphrases du type «de manière/façon + Adj ». Cette constatation incite à se demander pourquoi le système linguistique a choisi par pour former des séquences dont le sens est corrélable à la valeur d'un adverbe en -ment.

Un premier élément de réponse se trouve dans l'identité sémantique que nous attribuons à la préposition par, qui a trait, selon nous, à l'expression de la « modalité »valeur discursive assimilable à celle de la «manière de procéder » (cf. la question en Comment). Or précisément, les adverbes ou expressions adverbiales sont particulièrement dévolus, dans la langue, à cette fonction de véhiculer la modalité, le « comment».

Le deuxième élément de réponse, on pourrait le trouver dans le rôle même du processus du figement, tel que défini par S. Mejri (op. cit.) ; l'auteur démontre que ce phénomène assure le « renouvellement du lexique »- processus qu'il rapproche justement de celui de la dérivation en morphologie. En effet, pour former des adverbes, la langue dispose principalement du suffixe - ment qui vient se greffer sur des adjectifs exprimant un état ou une qualité pour renvoyer à la notion de «manière » (juste => justement ; formidable $=>$ formidablement ; tendre $=>$ tendrement ; long $=>$ longuement $;$ etc.) ; cependant, d'un côté, certains adjectifs ne sont pas compatibles avec ce suffixe (cf. bleu $=>$ *bleu(e)ment ; compliqué $=>$ *compliquément ; etc.), de l'autre, il n'y a pas que les adjectifs qui expriment des états ou des qualités : les $\mathrm{N}$ aussi peuvent exprimer les mêmes valeurs et l'on peut éprouver le besoin de présenter cette notion comme une «manière "; toutefois, dans le système linguistique, il n'est pas permis de dériver un adverbe en -ment, par exemple, à partir d'un nom ( $c$. bonbeur $=>$ *bonheureusement; contre $=>*^{*}$ contrement; exemple $=>*^{*}$ exemplement ; etc.). De fait, la préposition par est le moyen choisi par l'usage linguistique pour construire, à partir d'un nom, un syntagme dont la valeur est proche de celle véhiculée globalement par un adverbe : « la façon de procéder $»$.

Certes, on pourrait avoir parallèlement à certaines locutions en par (cf. par miracle, par erreur, par malheur, par euphémisme, par magie, par convention, etc.) des adverbes dérivés d'un adjectif apparenté morphologiquement à ces $\mathrm{N}$ : miraculeusement, erronément, malheureusement, euphémiquement, magiquement, conventionnellement, etc. ; mais, on s'apercevra que le sens change considérablement par rapport à ce que disent les locutions en par, ce qui constitue un argument de plus pour faire de par un meilleur candidat pour ce type de construction ${ }^{8}$. Comparons :

21. Il a par erreur attribué cet ouvrage à Rabelais.

22. Il a erronément attribué cet ouvrage à Rabelais.

\footnotetext{
${ }^{8}$ Une comparaison avec la préposition avec (qui a également des affinités avec l'expression de la "manière », dans par exemple: Il a accepté avec modestie $\approx I l$ a accepté modestement), montre que seule la préposition par peut se combiner avec ces $\mathrm{N}$ et produire la nuance de sens observée («modalité générique et contrastive »). La préposition avec, quant à elle, est soit impossible : *Il a attribué cet ouvrage à Rabelais avec erreur; *Elle est partie avec malheur, etc. soit donne un sens complètement différent à l'énoncé (proche de celui de l'adverbe en $-m e n t)$ : Il a agi avec prudence $(\approx$ «il était prudent/ Il a agi prudemment »).
} 
Par erreur veut dire à peu près " par inadvertance », " par mégarde »; cela oppose le procès à sa négation («il s'est trompé en disant cela; s'il n’avait pas dit cela, il n'y aurait pas eu d'erreur/c'est parce qu'il a dit cela qu'il y a erreur »), alors que erronément qualifie les propos : ils sont erronés, «ce qu'il a dit est une erreur». L'analyse est la même pour le couple par malheur (= «à cause de la malchance » $\approx$ 《par malchance ») versus malheureusement («l'événement lui-même rend le locuteur malheureux »). En (23) et (24) :

\section{Par malheur, elle est partie versus}

\section{Malheureusement, elle est partie.}

(23) est paraphrasable par «C'est la malchance qui est responsable de son départ / dans de meilleures circonstances, elle ne serait pas partie » versus (24) "C'est malheureux/Je suis malheureux, elle est partie ». De même, dire conventionnellement indique que " ce qui est dit est conventionnel », alors que dire par convention met davantage l'accent sur l'enjeu de l'aboutissement du procès (" on se conforme aux conventions »); dire euphémiquement, c'est «émettre des propos euphémiques, atténués", par opposition à par euphémisme qui signifie que «le résultat est obtenu par le biais d'un euphémisme, une atténuation »; apparaître magiquement («l'apparition est magique/extraordinaire ») versus par magie ("d'une manière que l'on ne peut expliquer »); miraculeusement (" extraordinairement, d'une façon remarquable ») versus par miracle («d'une façon inattendue et heureuse »). L'opposition entre la valeur propre de $\operatorname{par} N$ face à un $A d v$ en -ment apparait clairement dans les caractéristiques distributionnelles du couple par miracle/miraculeusement : ainsi, apparaître miraculeusement correspond à la tournure à verbe support faire une apparition miraculeuse, ce qui n'est pas le cas de apparaître par miracle; d'ailleurs la nominalisation précédée du verbe support faire ici n'est pas naturelle ??Faire une apparition par miracle.

Par conséquent, le rôle de par est très proche de celui que joue un suffixe dans la dérivation morphologique ; cette préposition permet de présenter un $\mathrm{N}$ donné sous l'angle d'une certaine «modalité » qui diffère, du point de vue du sens, de l'adverbe en -ment. On peut expliquer ce processus de formation suivant le phénomène de «transfert de catégorie » proposé par S. Mejri (op. cit.) pour les cas des N qui ne sont corrélables à des adjectifs (carte $=>{ }^{*}$ carteux $/{ }^{*}$ carté, ce qui explique la formation de la séquence figée adjectivale à la carte dans dîner à la carte).

Somme toute, la formation des unités polylexicales en par est imposée par deux types de besoin linguistique : le premier est que pour exprimer une valeur proche de la valeur d'un $\mathrm{Adv}$ en -ment à partir d'un $\mathrm{N}$, le processus de dérivation n'offre pas toujours cette possibilité, et le second est que les séquences du type $P A R N$ sont, de toute façon, nécessaires pour exprimer un certain rapport de «modalité contrastive» que l'Adv en -ment n'est pas en mesure d'exprimer, même quand la construction est possible (cf. par erreur versus erronément). 


\section{Conclusion générale}

Ainsi, différents indices montrent que les collocations en par prises en compte dans ce travail font preuve d'une certaine résistance à l'égard de certains tests syntaxiques de transformation qui sont de nature à spécifier le $N$ régi par la préposition par. Mais ce blocage n'est que partiel ; en effet, une approche compositionnelle reste possible, puisque les constituants de ces séquences conservent grosso modo leur sens, mais revêtent, en revanche, une certaine généricité, ce que corrobore le fait que, dans un grand nombre de ces locutions, on peut identifier la présence d'un article zéro. Cela conduit à parler, en l'occurrence, plutôt de «semi-figement»; on a affaire à des séquences dont le figement s'opère de façon «non oblique » - pour reprendre les termes de S. Mejri (2004) ; c'est-à-dire que le sens des composants n'est pas tout à fait opaque; les séquences admettent, malgré tout, une approche compositionnelle où l'on peut reconnaitre le signifié de chacun de ses constituants ${ }^{9}$. Ce phénomène de polylexicalité en par s'étend à certaines séquences dont le $\mathrm{N}$ relève de paradigmes variés (par amour, par jalousie, par pudeur, par prudence, par précaution, par peur, par courtoisie, par gentillesse, par méchanceté, par vengeance, par démagogie, par cupidité, par férocité, par réserve, par amitié, par courrier, par avion, par bateau, par fax, par téléphone, par télégramme, par jour, par semaine, par mois, par groupe, etc.); ces différentes collocations, quoique très productives (les $\mathrm{N} \mathrm{y}$ commutent les uns avec les autres), font preuve d'un blocage partiel vis-à-vis de certains tests de transformation (ajout d'un déterminant, différentes insertions, etc.). Cela est dû, selon nous, au fait que ces $\mathrm{N}$ perdent leur rapport de dénomination ou de désignation dès qu'ils sont associés à la préposition par. Ainsi, le $\mathrm{N}$ jour dans Il vient trois fois par jour n'admet ni l'actualisation (*Il vient trois fois par (ce + le $+u n+$ quelques + plusieurs) jour(s)), ni la pronominalisation (*Il vient par lui), ni l'anaphorisation (*Il vient trois fois par jour. ${ }^{*} \mathrm{Il} / \mathrm{Ce}$ jour était férie), ni le mouvement de $Q u$ (*Le jour par lequel il est venu trois fois), etc., ce qui est à relier à la valeur de par et ses affinités avec l'expression de la généricité. Par se présente ainsi comme un "générateur de polylexicalité ».

Cette tentative d'établir les fonctionnements linguistiques qui régissent la formation des collocations en par et d'en circonscrire le sens, la valeur grammaticale et le degré de figement peut aider - dans un souci d'exhaustivité - à tenir compte de ce type de séquences aussi bien dans les descriptions lexicographiques que dans l'enseignement du FLN, du FLS et du FLE. De même, les résultats obtenus ici peuvent constituer une matière de saisie informatique dans les différents projets de TALN (dictionnaires électroniques, traduction automatique, conception des bases de données textuelles, etc.). D'ailleurs, l'inventaire des expressions (semi-)figées en par que nous annexons à ce travail - et qui a fait l'objet d'un travail de vérification systématique - est une ébauche à ce type de projet : les séquences considérées dans cette étude manifestent des régularités linguistiques incontestables. Cet inventaire ( $c$. annexe) peut être complété par le recours aux mêmes critères linguistiques que nous nous sommes donnés dans le présent travail, comme il peut servir de modèle pour l'approche des autres collocations du français.

\footnotetext{
${ }^{9}$ Bien entendu, cette constatation n'est qu'une tendance générale; cela ne vaut pas, par exemple, pour quelques cas, comme Manger les pissenlits par la racine, Tirer le diable par la queue, etc. où l'on peut, a priori, identifier l'apport sémantique de par, mais non les autres composants, en tous cas, non en synchronie.
} 
La préposition par, génératrice de polylexicalité?

\section{ÉLÉMENTS BIBLIOGRAPHIQUES}

Anscombre, J.-C. (1990b), "Pourquoi un moulin à vent n'est pas un ventilateur ", Langue française 86, Paris, Larousse, pp. 103-125.

Gosselin, L. (1996), Sémantique de la temporalité en français, Louvain-la-Neuve, Duculot.

Gross, G. (1996), Les expressions figées en français. Noms composés et autres locutions, Paris, Ophrys.

Hamma, B. (2002), "Par exemple: l'expression de l'altérité dans l'acte d'exemplification », Revue de Sémantique et de Pragmatique, 15-16, pp. 155-181 (paru en 2004).

Hamma, B. (2004a), « Commencer et finir + par, des semi-auxiliaires non élus à l'unanimité ! Débuter, terminer et couronner + par, des candidats malheureux à l'« auxiliarité $» ! »$, actes de la Journée d'Etude, Didactique de la syntaxe : Le verbe dans tous ses états, actes coordonnés par C. Vaguer et B. Lavieu, Presses Universitaires de Namur, Coll. «Diptyque », pp. 95-115.

HAMmA, B. (2004b), «Sur quelques emplois du verbe «terminatif» finir : entre « semiauxiliaire » et « verbe libre », actes du Colloque de Nancy Sur la route du verbe (à paraitre).

HAMmA, B. (2005a), L'invariant sémantique de la préposition par à travers les distributions syntaxiques et lexicales, Thèse de doctorat, sous la direction de Danielle Leeman, Paris X Nanterre.

Hamma, B. (2005b), «Travail de corpus et modélisation des données : le cas des construc-

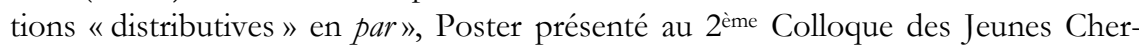
cheurs du Laboratoire MoDyCo à Paris X - Nanterre, Recueil des données en Sciences $d u$ langage et constitution de corpus.

Leeman, D. (1998), Les circonstants en question(s), Paris, Kimé.

MEJRI, S. (1997), "Structuration sémantique et variation des séquences figées », in S. Mejri (dir.), Le figement lexical. Descriptions linguistiques et structuration sémantique, Tunis, Publications de la Faculté des Lettres de la Manouba.

MEJRI, S. (2000b), «Figement et renouvellement du lexique : quand le processus détermine la dynamique du système », Le français moderne LXVIII, 1, pp. 41-62.

MEJRI, S. (2002), «Le figement lexical : nouvelles tendances », Cabiers de lexicologie 80 : 213 225.

MEJRI, S. (2003), «Le figement lexical », Cahiers de Lexicologie 82, pp. 23-39.

MejRI, S. (2004), «Les séquences figées adjectivales », in J. François (ed.), L'adjectif en français et à travers les langues, Bibliothèque de Syntaxe \& Sémantique, Caen, Presses Universitaires de Caen, pp. 403-412.

MELIS, L. (2003), La préposition en français, Paris, Ophrys.

MiLNER, J.-C. (1989), Introduction à une science du langage, Paris, Seuil.

\section{DiCTIONNAIRES CONSULTÉS}

PRLF : Robert, P. (1994), Petit Robert de Langue Française, Paris, Le Robert.

TLF : Trésor de la Langue Française (sur CD-Rom).

GReCO : Grand Robert \& Collins (sur CD-Rom). 


\section{ANNEXE \\ Polylexicalités en par (emplois représentatifs)}

Par A + B
Par accès
Par accident
Par acclamation
Par acquit de conscience
Par ailleurs
Par an
Par avance
Par bonheur
Par bonté d'âme
Par bouffées
Par calcul
Par chance
Par cour
Par complaisance
Par conséquent
Par considération pour
Par contenance
Par contraste
Par contre
Par contrecoup
Par contumace
Par convention
Par défaut
Par definition
Par degré(s)
Par éclairs
Par écrit
Par effluves
Par enchantement
Par endroit(s)
Par erreur
Par essence
Par étape(s)
Par euphémisme
Par excellence
Par exception
Par excès (math)
Par exemple
Par extraordinaire
Par force
Par babitude
Par basard
Par impossible
Par inadvertance
Par indivis

Par instants

Par intervalles

Par jeu

Par jour

Parl'absurde

Par l'opération du Saint-

esprit

Par la bande

Par la force

Par la force des choses

Par la grâce de Dieu

Par la peau des fesses

Par la raison que

Par la suite

Par la voie aérienne

Par la voie des airs

Par la voie maritime

Par la voie terrestre

Par le fait même

Par le fait que

Par le fer et le feu

Par le menu

Par le monde

Par le passé

Par le plus grands des

hasards

Par le siège (accouche-

ment)

Par le vide

Par le(s) temps qui

cour(en)t

Par ma foi

Par magie

Par malchance

Par malheur

Par manque de

bol/chance

Par mégarde

Par mésaventure

Par métaphore

Par miracle

Par moment(s)

Par monosyllabe

Par nature

Par occasion

Par ondes
Par opposition à

Par ordre

Par oü-dire

Parpaliers

Parparenthèse

Parpénitence

Parpériodes

Parpersonnes interposées

Parpitié

Par préférence

Parprincipe

Parprocuration

Par raccroc

Par retour du courrier

Par ricochet

Par saccade(s)

Par sauts

Par secousse

Par ses propres moyens

Par suite

Par surcroît

Par terre

Par tête

Par tête de pipe

Par tous les diables

Par tous les moyens

Par tous les temps

Par tradition

Par trop curieux

Par trop important

Par un beureux concours

des circonstances

Par un malheureux

concours des circonstances

Par une raison bien simple

Par vagues

Par voie aérienne

Par voie de conséquence

Par voie de succession

Par voie biérarchique

Par voie maritime

Par voie orale

Par voie postale

Par voie terrestre

Par voies et chemins. 